\title{
Terahertz spectroscopy of the CHD radical $\left(\widetilde{X}^{3} A^{\prime \prime}\right)$
}

\author{
H. Ozeki ${ }^{1}$, S. Bailleux ${ }^{2}$, and G. Wlodarczak ${ }^{2}$ \\ 1 Department of Environmental Science, Faculty of Science, Toho University, 2-2-1 Miyama, Funabashi 274-8510, Japan \\ e-mail: ozeki@env.sci.toho-u.ac.jp \\ 2 Laboratoire de Physique des Lasers, Atomes et Molécules, UMR CNRS 8523, CERLA, Université Lille 1, \\ 59655 Villeneuve d'Ascq Cedex, France \\ e-mail: stephane.bailleux@univ-lille1.fr
}

Received 2 November 2010 / Accepted 26 November 2010

\begin{abstract}
Context. Pure rotational spectra in the field-free condition of the $\mathrm{CH}_{2}$ and $\mathrm{CD}_{2}$ isotopologues of the methylene radical have already been reported, whereas only six rotational transitions of the mono-deuterated species, CHD, detected by FIR-LMR spectroscopy have been.

Aims. Spectra of the mono-deuterated methylene radical in its ground electronic state $\left(\widetilde{X}^{3} A^{\prime \prime}\right)$ were observed in the field-free condition for the first time in the frequency region below $1 \mathrm{THz}$.

Methods. CHD was generated in a positive column discharge in a $\mathrm{CH}_{2} \mathrm{D}_{2}-\mathrm{Ar}$ mixture cooled with liquid nitrogen.

Results. Two rotational transitions, each split into three fine-structure components, were measured. The $B$ and $C$ rotational constants, the $\epsilon_{b b}$ and $\epsilon_{c c}$ spin-rotation constants, and the $\beta$ spin-spin constant were revised.

Conclusions. The reported frequencies are suitable for astronomical searches.
\end{abstract}

Key words. line: identification - molecular data - ISM: molecules - submillimetre: ISM

\section{Introduction}

The methylene radical, $\mathrm{CH}_{2}$, is known as an interstellar molecule that has been detected towards hot cores in dense interstellar clouds (Hollis et al. 1995; Polehampton et al. 2005). In the gas phase chemical models of both dense and diffuse molecular clouds, the methylene radical is thought to be one of the fundamental molecules of the carbon chemistry, and it is produced primarily by dissociative recombination of the methyl ion, $\mathrm{CH}_{3}^{+}$(Herbst 2001; Vejby-Christensen et al. 1997). Recently tentative detection of the mono-deuterated methyl ion, $\mathrm{CH}_{2} \mathrm{D}^{+}$have been reported toward an infrared source in the vicinity of Orion (Lis et al. 2009), along with its supporting laboratory spectroscopic measurements (Amano 2010; Gärtner et al. 2010). Monodeuterated methylene CHD can be produced from this ion or its counterpart ion, $\mathrm{CHD}_{2}^{+}$, by dissociative recombination with an electron as shown in the chemical reactions:

$$
\begin{aligned}
& \mathrm{CH}_{2} \mathrm{D}^{+}+\mathrm{e}^{-} \rightarrow \mathrm{CHD}+\mathrm{H} \text { or } \mathrm{CH}_{2}+\mathrm{D}, \\
& \mathrm{CHD}_{2}^{+}+\mathrm{e}^{-} \rightarrow \mathrm{CHD}+\mathrm{D} \text { or } \mathrm{CD}_{2}+\mathrm{H} .
\end{aligned}
$$

Thus, both $\mathrm{CHD}$ and $\mathrm{CD}_{2}$ can be observed in warm interstellar clouds, where the deuterium fractionation process is important (see Turner 2001; Roueff 2007; Parise 2009). Precise laboratory reference data are therefore desirable for such a radioastronomical observation of these molecules.

More than half a century has passed since the pioneering spectroscopic work on the methylene radicals, $\mathrm{CH}_{2}$ and its deuterated isotopomers by Herzberg et al. (1959, 1971). However, it is still an ongoing project to characterize or understand the nature of this species by spectroscopy in various wavelength regions. This difficulty arises from the unique chemical and physical properties of the molecule. The methylene radical is the prototype of the "carbene" and is known to be kinetically a very unstable species (Moss \& Jones, Jr. 1975). Therefore this short-lived molecule is very hard to generate in concentrations that are high enough for carrying out spectroscopic studies in laboratory conditions. In the ground electronic state, the $\mathrm{CH}_{2}$ molecule possesses ${ }^{3} B_{1}$ symmetry with a bent and exceedingly floppy molecular structure. The latter characteristic leads to the breakdown of the Watson model for this species. Such a complicated situation has stimulated many theoretical studies on $\mathrm{CH}_{2}$, and various models have been proposed in order to represent its bending potential (see Bunker \& Landsberg 1977; Bunker \& Jensen 1983; Jensen et al. 1982; Bunker et al. 1986; Jensen 1988). The fidelity of these theoretical models can be validated by comparative studies of the energy levels of methylene and its deuterated analogues obtained by high-resolution molecular spectroscopy.

High-resolution spectroscopy of the methylene radical has been conducted mainly by infrared/far-infrared laser magnetic resonance spectroscopy (IR/FIR-LMR) or infrared diode laser spectroscopy (Bunker 1985). Thanks to these extensive works in mid 70's to early 80 's, pure rotational transitions frequencies for $\mathrm{CH}_{2}$ and $\mathrm{CD}_{2}$ can be predicted with the accuracy of less than several tens of $\mathrm{MHz}$, and laboratory microwave spectra of $\mathrm{CH}_{2}$ and $\mathrm{CD}_{2}$ were successfully observed (Lovas 1983; Ozeki \& Saito 1995, 1996). In the best-studied case of $\mathrm{CH}_{2}$, six rotational transitions having $K_{a}=0,1$ have been observed up to almost $2 \mathrm{THz}$ with microwave spectroscopic precision (Michael et al. 2003; Brünken et al. 2004, 2005). Since $b$-type transitions are allowed, those measured near 1 and $2 \mathrm{THz}$ involve $N=1,2$ and include the $1_{10} \leftarrow 1_{01}$ ground-state transition of para- $\mathrm{CH}_{2}$ (see the definition of ortho- and para- $\mathrm{CH}_{2}$ in the results and discussion section), while the ones measured in the lower frequency region correspond to higher rotational quantum numbers with 
energy levels of several hundred Kelvin. The inadequacy of a Watson-type Hamiltonian for fitting spectra of methylene radical also led Brünken et al. (2005) to employ a Hamiltonian based on Euler functions to successfully carry out the global analysis of the experimental spectrum of $\mathrm{CH}_{2}$. The number of observed lines is, however, still limited due to its lightness and the fact that $b$-type asymmetric rotor selection rules apply. As for $\mathrm{CD}_{2}$, only four rotational transitions below $600 \mathrm{GHz}$ have been reported so far by laboratory microwave spectroscopy (Ozeki \& Saito 1996). To our knowledge, no field-free high-resolution spectrum has been reported for the CHD radical. Nolte et al. (1994) published FIR-LMR spectroscopy of the CHD radical in the ground electronic state. An insufficient number of observed spectral lines (six rotational transitions) meant that their proposed set of molecular constants was a composite of the ones derived both experimentally and theoretically (Bunker et al. 1983). As a consequence the energy levels of CHD are not as reliable as those of $\mathrm{CH}_{2}$ and $\mathrm{CD}_{2}$, which presumably makes it hard to achieve spectral measurement of this species. We report in the present paper field-free, pure rotational spectra of the CHD radical up to the terahertz frequency region with an accuracy the same order as conventional microwave spectroscopy.

\section{Experiment}

The spectrometer used in this experiment has been described extensively elsewhere (Bailleux et al. 2002). Submillimetre-wave and terahertz radiations in the frequency range $0.55-0.97 \mathrm{THz}$ were provided by two phase-locked backward-wave oscillators (Istok company, Russia). Emitted power at observed transition frequencies was in the $\mathrm{mW}$ range. A liquid-helium-cooled $\mathrm{InSb}$ bolometer (QMC Instruments) was used as detector. Source modulation at $5 \mathrm{kHz}$ and lock-in detection at twice the modulation frequency was employed for improved sensitivity, resulting in a second-derivative line shape. A positive column discharge in various isotopologues of methane used as precursors, $\mathrm{CH}_{2} \mathrm{D}_{2}$ and $\mathrm{CD}_{4}$ (Cambridge Isotope Laboratories Inc., USA), was setup. The absorption cell was a $2 \mathrm{~m}$ long, $5 \mathrm{~cm}$ inner diameter, double-jacketed Pyrex tube, allowing liquid-nitrogen flow through the outer jacket to cool the plasma. The cell was equipped with a solenoid coil for creating an axial magnetic field to ensure that the lines are paramagnetic by on/off measurements. The precursor was pre-mixed with argon used as buffer gas before it entered the absorption cell's inlet for in situ production of reactive species. In addition to the above-mentioned precursors, $\mathrm{CH}_{4}$ was also available in order to check for the chemistry of the reactions that occur in the absorption cell.

\section{Spectroscopic measurements}

We first optimized the experimental conditions to produce $\mathrm{CH}_{2}$ as trial species using $\mathrm{CH}_{4}$ as precursor. For this purpose, reported rotational lines below $600 \mathrm{GHz}$ were observed. The conditions were further confirmed by the observation of the di-deuterated analogue, $\mathrm{CD}_{2}$, using $\mathrm{CD}_{4}$ as precursor. We found that the optimum discharge current was in the range 20-30 mA and that the partial pressures (measured downstream at room temperature) giving the best signal-to-noise ratio were 13 and 2.5 mTorr for $\mathrm{Ar}$ and $\mathrm{CH}_{4}$ or $\mathrm{CD}_{4}$, respectively. The outer jacket of the cell partially filled with liquid nitrogen $(\sim 30 \%$ of the maximum liquid nitrogen load) resulted in an increase in the intensity of the signals. Using these conditions, we have successfully observed the as yet unreported three fine-structure components of the
$N_{K_{a} K_{c}}=7_{07} \leftarrow 6_{16}$ rotational transition of $\mathrm{CD}_{2}$ near $799 \mathrm{GHz}$, which will be published elsewhere with additional lines measured, if possible. Optimization of the conditions also allowed us to gauge both the production efficiency and the line intensity we could expect for the mono-deuterated methylene radical. Indeed, our method of production differs markedly from the one reported in previous studies, continuous glow discharge in ketene. As a result, our production efficiency, although lower than the one obtained with the discharge in ketene, was satisfactory to expect the detection of rotational transitions of CHD.

We afterwards guided our search for the $N_{K_{a} K_{c}}=7_{07} \leftarrow 6_{16}$ rotational transition of $\mathrm{CHD}, \mathrm{CH}_{2} \mathrm{D}_{2}$ serving as precursor. The three fine-structure components were expected to occur near 944.5 GHz, based on the predictions made using the molecular constants provided by the FIR-LMR work (Nolte et al. 1994). A careful search of $2 \mathrm{GHz}$ around the predicted frequencies using the same experimental conditions as for $\mathrm{CH}_{2}$ and $\mathrm{CD}_{2}$ showed very clean spectra. A limited number of unidentified lines were detected, but all were found to belong to diamagnetic species. We thus decided to extend the search region further, proceeding toward the high frequencies. We detected one set of three paramagnetic lines within $512 \mathrm{MHz}$. We checked the chemistry to produce these lines and found that they were not observable when $\mathrm{CD}_{4}$ or $\mathrm{CH}_{4}$ were used as precursors instead of $\mathrm{CH}_{2} \mathrm{D}_{2}$. However, they could be detected when $\mathrm{CD}_{4}$ and $\mathrm{CH}_{4}$ were introduced simultaneously, with a signal-to-noise ratio about five times weaker than with $\mathrm{CH}_{2} \mathrm{D}_{2}$. These chemical tests showed that the newly observed species contains both $\mathrm{H}$ and $\mathrm{D}$ atoms. Convinced that this species was the monodeuterated methylene radical, we decided to detect the $N_{K_{a} K_{c}}=6_{06} \leftarrow 5_{15}$ rotational transition predicted around $580.3 \mathrm{GHz}$ and started to scan upwards. We subsequently discovered another set of three paramagnetic transitions above $581 \mathrm{GHz}$ that showed identical behaviour with the chemical tests. The detection of two additional rotational transitions, the $N_{K_{a} K_{c}}=5_{05} \leftarrow 4_{14}$ and the $N_{K_{a} K_{c}}=1_{11} \leftarrow 2_{02}$, predicted near 221 and $821 \mathrm{GHz}$, respectively, was attempted. It is likely that the lack of accurate predictions (within $100 \mathrm{MHz}$ ) and their weaker intensity, especially for the $N_{K_{a} K_{c}}=1_{11} \leftarrow 2_{02}, J=2 \leftarrow 3$ fine-structure component that is expected to be split into several hyperfine-structure components, prevented us from detecting them.

\section{Results and discussion}

Although the number of observed lines is limited, we are confident that identification of the CHD radical in its ground electronic state $\left(\widetilde{X}^{3} A^{\prime \prime}\right)$ is secure. Three fine-structure components for each rotational transition were detected, as expected for a species in a triplet ground electronic state. Wide regions were scanned (several GHz) around the predictions, and only the transition frequencies reported in Table 1 showed paramagnetic behaviour. The chemistry for all six lines was carefully checked, proving that they belong to a species that contains both $\mathrm{H}$ and $\mathrm{D}$ atoms. As for $\mathrm{CH}_{2}$ and $\mathrm{CD}_{2}$, all fine-structure components were found to be very sensitive to the magnetic field applied: a magnetic field in the range 2-50 Gauss was sufficient to observe the disappearance of the lines, the $F_{3}$ and $F_{2}$ components being the most and least sensitive, respectively. Similar to the spectrum of $\mathrm{CD}_{2}$ at $799 \mathrm{GHz}$, the $F_{2}$ component was detected with stronger signal-to-noise ratio than the $F_{1}$ and $F_{3}$ components. These observations are in accordance with the prediction that includes the hyperfine-structure due to $\mathrm{H}$ and $\mathrm{D}$ nuclei since all hyperfine-structure components are overlapped on a single line only for the $F_{2}$ component. An example of the 
Table 1. Observed rotational transitions frequencies of $\operatorname{CHD}\left(\widetilde{X}^{3} A^{\prime \prime}\right)$, in MHz.

\begin{tabular}{cccc}
\hline \hline Transition & Component $^{a}$ & $v_{\text {obs }}{ }^{b}$ & $v_{\text {obs }}-v_{\text {calc }}$ \\
\hline $6_{06} \leftarrow 5_{15}$ & $F_{1}$ & $581021.489(60)^{c}$ & 0.046 \\
& $F_{2}$ & $581506.679(20)$ & -0.012 \\
& $F_{3}$ & $581580.606(50)$ & 0.040 \\
$7_{07} \leftarrow 6_{16}$ & $F_{1}$ & $946609.805(40)$ & -0.022 \\
& $F_{2}$ & $946800.636(25)$ & 0.019 \\
& $F_{3}$ & $947121.971(60)$ & -0.060 \\
\hline
\end{tabular}

Notes. The standard deviation of the fit is $38 \mathrm{kHz}$. Calculated frequencies were obtained from molecular constants given in Table 2. (a) $\Delta J=\Delta N=1$ fine-structure components. For the upper and lower energy levels, $F_{1}: J=N+1, F_{2}: J=N, F_{3}: J=N-1{ }^{(b)}$ Values in parentheses are $1 \sigma$ experimental error on the last digits. ${ }^{(c)}$ Hyperfinestructure for this line was partly resolved as a doublet, and the average of the two observed transition frequencies was used in the least-squares analysis.

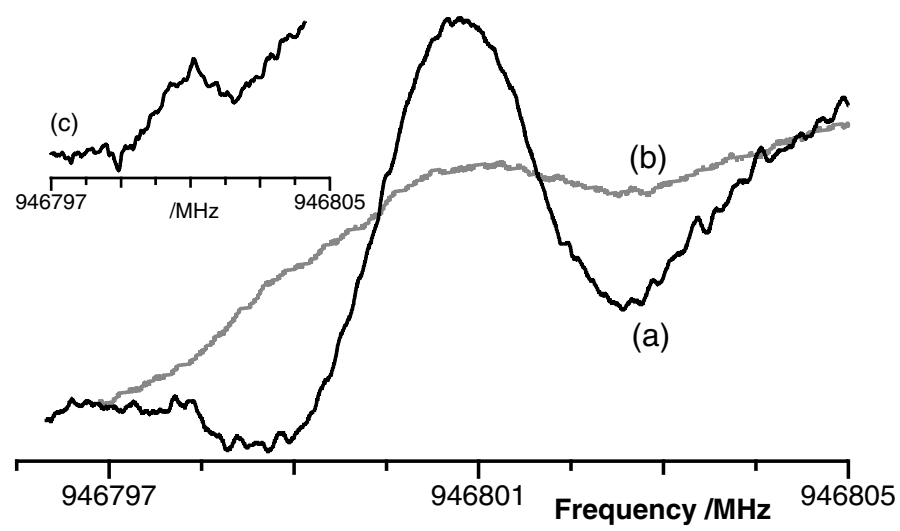

Fig. 1. Observed spectra of the $N_{K_{a} K_{c}}=7_{07} \leftarrow 6_{16}, J=7 \leftarrow 6\left(F_{2}\right.$ component) rotational transition of the $\mathrm{CHD}$ radical produced in de glow discharge $(\sim 20 \mathrm{~mA})$ using $\mathrm{CH}_{2} \mathrm{D}_{2}$ as precursor recorded a) without magnetic field applied and $\mathbf{b}$ ) with a magnetic field of only 15 Gauss applied. Inset c): same transition detected using $\mathrm{CH}_{4}$ and $\mathrm{CD}_{4}$ as precursors, about five times less intense. These spectra were recorded by integrating 36 scans with $0.5 \mathrm{~Hz}$ repetition rate and $10 \mathrm{~ms}$ time constant of the lock-in amplifier.

$N_{K_{a} K_{c}}=7_{07} \leftarrow 6_{16}, J=7 \leftarrow 6\left(F_{2}\right.$ component $)$ recorded near $946801 \mathrm{MHz}$ is depicted in Fig. 1.

In the present study, no hyperfine-structure due to $\mathrm{H}$ and D nuclei could be observed for these high $N$ rotational transitions, except for the $F_{1}$ component of the $6_{06} \leftarrow 5_{15}$ rotational transition, which appeared as a weak, partly resolved doublet with a frequency gap of 1.34(12) MHz. This value was obtained from a nonlinear line profile analysis for individual components using IGOR Pro (wavemetrics). The line frequencies and the peak intensities for this doublet were adjusted by assuming a second derivative Voigt line shape with the same linewidth for both hyperfine-structure components. This result is consistent with our preliminary prediction of the hyperfine-structures by assuming that the hyperfine interaction constants for $\mathrm{H}$ and $\mathrm{D}$ nuclei are the same as those of $\mathrm{CH}_{2}$ and $\mathrm{CD}_{2}$, respectively. The calculated hyperfine pattern for these high $N$ rotational quantum numbers shows that the $F_{2}$ components should be observed as a singlet, while the $F_{1}$ and $F_{3}$ components should each appear as a doublet with a frequency gap of less than 3.0 and $2.5 \mathrm{MHz}$,
Table 2. Molecular constants for $\mathrm{CHD}\left(\widetilde{X}^{3} A^{\prime \prime}\right)$, in MHz.

\begin{tabular}{lcc}
\hline \hline Constant & Present study & FIR LMR work $^{a}$ \\
\hline$B$ & $170344.0470(28)$ & $170233.7(21)$ \\
$C$ & $148139.235(32)$ & $149100.6(21)$ \\
$\epsilon_{b b}$ & $-112.66(18)$ & -105.527 \\
$\epsilon_{c c}$ & $-110.93(50)$ & -83.342 \\
$\beta$ & $1339.584(75)$ & $1382.0(75)$ \\
\hline
\end{tabular}

Notes. Only constants determined in this preliminary study are reported. All other molecular constants have been fixed to their values reported in Table IV from Reference 1. ${ }^{(a)}$ Reference 1. Values originally reported in $\mathrm{cm}^{-1}$ were converted to $\mathrm{MHz}$.

References. (1) Nolte et al. (1994).

respectively. It should also be noted that, at these high frequencies, the Doppler broadening of the lines prevents the observation of the hyperfine-structure. The observed transition frequencies (Table 1) were subjected to least-squares analysis (which does not include hyperfine constants) using Pickett's program SPFIT (Pickett 1991). The rotational constants $B$ and $C$, the electron spin-rotation constants $\epsilon_{b b}$ and $\epsilon_{c c}$, and the electron spin-electron spin constant $\beta$ were the adjustable parameters. A least-squares analysis using the combinations of constants $A-\frac{1}{2}(B+C), \frac{1}{2}(B+C), \epsilon_{a a}-\frac{1}{2}\left(\epsilon_{b b}+\epsilon_{c c}\right)$, and $\frac{1}{2}\left(\epsilon_{b b}+\epsilon_{c c}\right)$, in addition to $\beta$, resulted in a fit with a standard deviation of $\sim 0.7 \mathrm{MHz}$. The determined constants are provided in Table 2, together with their values obtained from the previous FIR-LMR study for comparison. We checked that no significant correlation occurred between the floated parameters.

Obviously, the rotational constants determined in the present study do not fit the quoted error range of the ones obtained by the previous FIR-LMR work. This is true in particular for the $C$ constant. In contrast, the fine-structure parameters of the two studies compare much better. Indeed, the frequency differences between two successive fine-structure components of the $N=6 \leftarrow 5$ and $7 \leftarrow 6$ rotational transitions predicted from the LMR work can be compared with those obtained from our measurements. The frequency differences from the LMR work, given in $\mathrm{MHz}$ as $\left(F_{2}-F_{1}, F_{3}-F_{2}\right)$ are $(460,112)$ and $(168,363)$ for the $N=6 \leftarrow 5$ and $7 \leftarrow 6$ transitions, respectively. The corresponding differences calculated from Table 1 are $(485,74)$ and (191, 321).

The set of molecular constants reported by Nolte et al. (1994) consisted of eight adjusted and nine fixed parameters for both the rotational and spin-rotational parts. The fitted parameters were determined by a limited number of measured lines and theoretically calculated energy levels, while eight out of the nine fixed parameters were constrained to the average of their values for $\mathrm{CH}_{2}$ and $\mathrm{CD}_{2}$. The estimated errors (10 to $15 \mathrm{MHz}$ ) for their measured lines are almost certainly underestimated, as they stated. Considering these facts, it is not surprising that the transition frequencies observed in the present study are found shifted by as much as 1 to $2 \mathrm{GHz}$ from the FIR-LMR predicted values. This shows a striking contrast to the cases of spectroscopy of $\mathrm{CH}_{2}$ and $\mathrm{CD}_{2}$, as FIR-LMR data for those species were quite reliable. Our constants will undoubtedly evolve as further rotational transitions of this species will be measured in the future. Since the errors on our constants are model-dependent, they are underestimated as well. Our reported frequencies can be useful as the precise laboratory reference data for astronomical observation, though the upper levels of the observed transitions are 
relatively high $\left(220-300 \mathrm{~cm}^{-1}\right.$, corresponding to temperatures of 300-400 K).

Finally, the ground-state rotational transition deserves a few comments. There are no two equivalent nuclei in CHD. Thus no spin statistics have to be obeyed, and only one ground-state transition for this species exists, the $1_{11} \leftarrow 0_{00}$. The rest frequencies of this transition, not taking the fine and hyperfine interactions into account, is predicted to occur near $1773 \mathrm{GHz}$ and $1774 \mathrm{GHz}$, based on our results and on the LMR work, respectively. In contrast, $\mathrm{CH}_{2}$ and $\mathrm{CD}_{2}$ have ${ }^{3} B_{1}$ as a ground state and should follow the Fermi-Dirac and Bose-Einstein spin statistics, respectively. Therefore ortho- and para-states, which are represented by even and odd values of $K_{a}+K_{c}$, respectively, coexist. The ground-state transitions of the two ortho-species lie near 2.35 and $1.23 \mathrm{THz}$, and in addition, two fundamental transitions of the para-species occur, namely the $1_{10} \leftarrow 1_{01}$ and the $2_{12} \leftarrow 1_{01}$. Their lower energy level is $15.1 \mathrm{~cm}^{-1}$ and $7.4 \mathrm{~cm}^{-1}$ above the ground in the case of $\mathrm{CH}_{2}$ and $\mathrm{CD}_{2}$, respectively. None of these ground-state transitions has yet been measured with microwave accuracy.

It is also worthwhile mentioning that the $7_{07} \leftarrow 6_{16}$ transition at $0.94 \mathrm{THz}$ lies in the centre of one of the "atmospheric windows" around $1 \mathrm{THz}$ (Matsushita et al. 2000), through which astronomical observations are feasible from ground-based observatories.

\section{Conclusions}

The two strongest field-free pure rotational transitions of the CHD radical in the electronic ground state $\left(\widetilde{X}^{3} A^{\prime \prime}\right)$ have been successfully observed below $1 \mathrm{THz}$ for the first time. It is necessary to increase the CHD production efficiency and/or the detection sensitivity of the spectrometer for further detection of the unobserved lines below or above $1 \mathrm{THz}$. We intend in the near feature to carry out such measurements using the "conventional" method, that is, in situ flash pyrolysis of partially deuterated diketene (to generate partially deuterated ketene) combined with continuous glow discharge.

Acknowledgements. The National French Programme "Physique et Chimie du Milieu Interstellaire" (PCMI) and the French National Research Agency
(ANR-08-BLAN-0225-01-02-03-04 "FORCOMS") are acknowledged for their support. H.O. gratefully acknowledges Lille 1 University for supporting his stay as an invited professor for two months.

\section{References}

Amano, T. 2010, A\&A, 516, L4

Bailleux, S., Bogey, M., Demuynck, C., Liu, Y., \& Walters, A. 2002, J. Mol. Spectrosc., 216, 465

Brünken, S., Michael, E., Lewen, F., et al. 2004, Can. J. Chem., 82, 676

Brünken, S., Müller, H. S. P., Lewen, F., \& Giesen, T. F. 2005, J. Chem. Phys., 123,164315

Bunker, P. R. 1985, in Comparison of Ab Initio Quantum Chemistry with Experiment for Small Molecules, ed. R. J. Bartlett (Dordrecht, Holland: D. Reidel Publishing Company), and references therein

Bunker, P. R., \& Jensen, P. 1983, J. Chem. Phys., 79, 1224

Bunker, P. R., \& Landsberg, B. M. 1977, J. Mol. Spectrosc., 67, 374

Bunker, P. R., Sears, T. J., McKellar, A. R. W., Evenson, K. M., \& Lovas, F. J. 1983, J. Chem. Phys.,79, 1211

Bunker, P. R., Jensen, P., Kraemer, W. P., \& Beardsworth, R. 1986, J. Chem. Phys., 85, 3724

Gärtner, S., Krieg, J., Klemann, A., Asvany, O., \& Schlemmer, S. 2010, A\&A, 516, L3

Herbst, E. 2001, Chem. Soc. Rev., 30, 168

Herzberg, G., \& Johns, J. W. C. 1971, J. Chem. Phys., 54, 2276

Herzberg, G., \& Shoosmith, J. 1959, Nature, 183, 1801

Hollis, J. M., Jewell, P. R., \& Lovas, F. J. 1995, ApJ, 438, 259

Jensen, P. 1988, J. Mol. Spectrosc., 128, 478

Jensen, P., Bunker, P. R., \& Hoy, A. R. 1982, J. Chem. Phys., 77, 5370

Lis, D. C., Goldsmith, P. F., Bergin, E. A., et al. 2009, in Submillimeter Astrophysics and Technology, ASP Conf. Ser., 417, 23

Lovas, F. J., Suenram, R. D., \& Evenson, K. M. 1983, ApJ, 267, L131

Matsushita, S., Matsuo, H., Sakamoto, A., \& Pardo, J. R. 2000, Proc. SPIE, 4015, 378

Michael, E. A., Lewen, F., Winnewisser, G., et al. 2003, ApJ, 596, 1356

Moss, R. A., \& Jones Jr., M. 1975, in Carbenes (New York: Wiley-Interscience), 2

Nolte, J., Temps, F., Wagner, H. Gg., Wolf, M., \& Sears, T. J. 1994, J. Chem. Phys., 100, 8706

Ozeki, H., \& Saito, S. 1995, ApJ, 451, L97

Ozeki, H., \& Saito, S. 1996, J. Chem. Phys., 104, 2167

Parise, B., Leurini, S., Schilke, P., et al. 2009, A\&A, 508, 737

Pickett, H. M. 1991, J. Mol. Spectrosc., 148, 371

Polehampton, E. T., Menten, K. M., Brünken, S., Winnewisser, G., \& Baluteau, J. P. 2005, A\&A, 431, 203

Roueff, E., Parise, B., \& Herbst, E. 2007, A\&A, 464, 245

Turner, B. E. 2001, ApJS, 136, 579

Vejby-Christensen, L., Andersen, L. H., Heber, O., et al. 1997, ApJ, 1997, 483, 531 